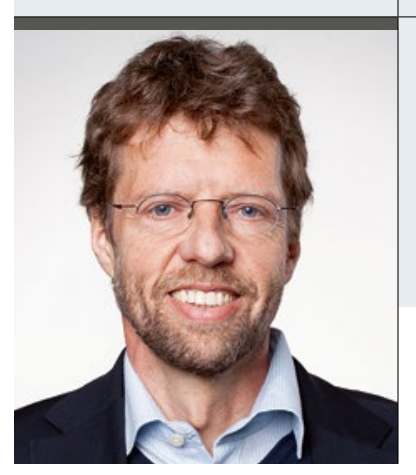

„Wenn die Regeln des IQWiG schon 1990 gegolten hätten, wäre

in Deutschland keines der sogenannten neuen Antiepileptika

auf dem Markt geblieben ..."

\title{
AMNOG und die Auswirkungen
}

$\mathrm{M}$ it Wirkung zum 1. Januar 2011 ist das Gesetz zur Neuordnung des Arzneimittelmarktes (AMNOG) nach $₫ 35$ a SGB V in Kraft getreten. Es regelt unter anderem die Preisbildung für neu zugelassene Arzneimittel. Der Gemeinsame Bundesausschuss (G-BA) legt anhand der frühen Nutzenbewertung des Instituts für Qualität und Wirtschaftlichkeit im Gesundheitswesen (IQWiG) den Zusatznutzen neu in den Markt eingeführter Arzneimittel fest. Bevor der G-BA einen Beschluss fasst, wird ein formalisiertes Verfahren durchgeführt, mit einer unlesbaren Menge an $\mathrm{Pa}$ pieren, Dossiers und Entgegnungen, nachzulesen im Detail auf der Webseite des G-BA [www.g-ba.de].

Insgesamt 144 Nutzenbewertungen konnte der G-BA seither abschließen. Dabei wiesen 81 der bewerteten Wirkstoffe einen Zusatznutzen auf. Bei zwei Wirkstoffen wurde ein erheblicher, bei 30 ein beträchtlicher, bei 33 ein geringer und bei 16 Wirkstoffen ein nicht quantifizierbarer Zusatznutzen festgestellt. Die Nutzenkategorie „erheblich“ wurde im Berichtsjahr 2015 zum ersten Mal vergeben. Bei 63 Nutzenbewertungen war kein Zusatznutzen belegbar. Davon wurden vier Arzneimittel sofort in eine Festbetragsgruppe eingeordnet, weil sie pharmakologisch-therapeutisch vergleichbar mit schon am Markt befindlichen Festbetragsarzneimitteln waren.

\section{Antiepileptika auf dem Prüfstand}

Interessanterweise wurden vor allem ZNS-aktive Wirkstoffe wie Antiepileptika (AE), Antidepressiva und Antipsychotika, aber auch Diabetes mellitusMedikamente ohne Zusatznutzen beurteilt. Das Hauptproblem des Verfahrens besteht darin, dass die notwendigen Zulassungsstudien für die Einführung eines neuen Wirkstoffes placebokontrollierte Studien als Maßstab festlegen, so wird es von der amerikanischen Zulassungsbehörde FDA und von der europäischen Behörde (EMA) gefordert. Nach Meinung nahezu aller Fachgesellschaften ist es derzeit im Rahmen des stark formalisierten Bewertungsprozesses des AMMOG, das die Zusammen- arbeit mit Fachgesellschaften nur nachrangig vorsieht, nicht verlässlich möglich, den Zusatznutzen neuer Medikamente reliabel und valide einzuschätzen. Versuche der eigenen Fachgesellschaft (Deutsche Gesellschaft für Epileptologie, DGfE) den Mitgliedern des G-BA und des IQWiG die Problematik bei solchen ZNS-aktiven Substanzen wie AE zu vermitteln, misslangen regelmäßig. So ist also mit Brivaracetam das dritte AE in Serie daran gescheitert, einen Zusatznutzen attestiert zu bekommen. Retigabin ist 2012 als sehr innovatives Medikament (Kalium-Auswärtsstrom-Hemmer) auf den Markt gekommen, wird aber ab 2017 komplett vom europäischen Markt genommen, auch wenn es gar nicht so wenig Patienten gibt, die davon profitieren. Perampanel, ein selektiver, nicht kompetitiver Antagonist an AMPA-Rezeptoren, ebenfalls als innovativ einzuschätzen, der sowohl bei fokalen wie generalisierten Epilepsien wirkt (und zugelassen ist), wurde vom deutschen Markt zurückgezogen, da der Hersteller Eisai hier keine Chance auf einen rentablen Preis sah. In Deutschland wird es jetzt über die internationale Apotheke bestellt, vorab muss aber eine Zusage der Krankenkasse vorliegen, die die Verordnung akzeptiert. Schließlich wurde am 4. August 2016 vom G-BA auch dem neuen AE Brivaracetam der Zusatznutzen aberkannt [www.akdae.de/Stellungnahmen/AMNOG]. Betroffene, oft behinderte Patienten mit aktiver Epilepsie werden solche neuen Substanzen auf Dauer ohnehin nur akzeptieren, wenn sie verträglich sind und einen individuellen Nutzen haben. Der G-BA-Entscheid wird nun dazu führen, dass medikamentöse Innovationen im ZNS-Bereich in Deutschland in den nächsten Jahren nicht mehr zu erwarten sind und damit auch keine Therapieoptionen mehr für diese Patientengruppe.

Epilepsien stellen für Betroffene, Angehörige und Gesellschaft eine erhebliche Belastung dar. Bei mehr als drei Viertel der Patienten erfolgt die Therapie mit Valproat, Carbamazepin, Lamotrigin oder Levetiracetam. Bei therapierefraktären Patienten ist 


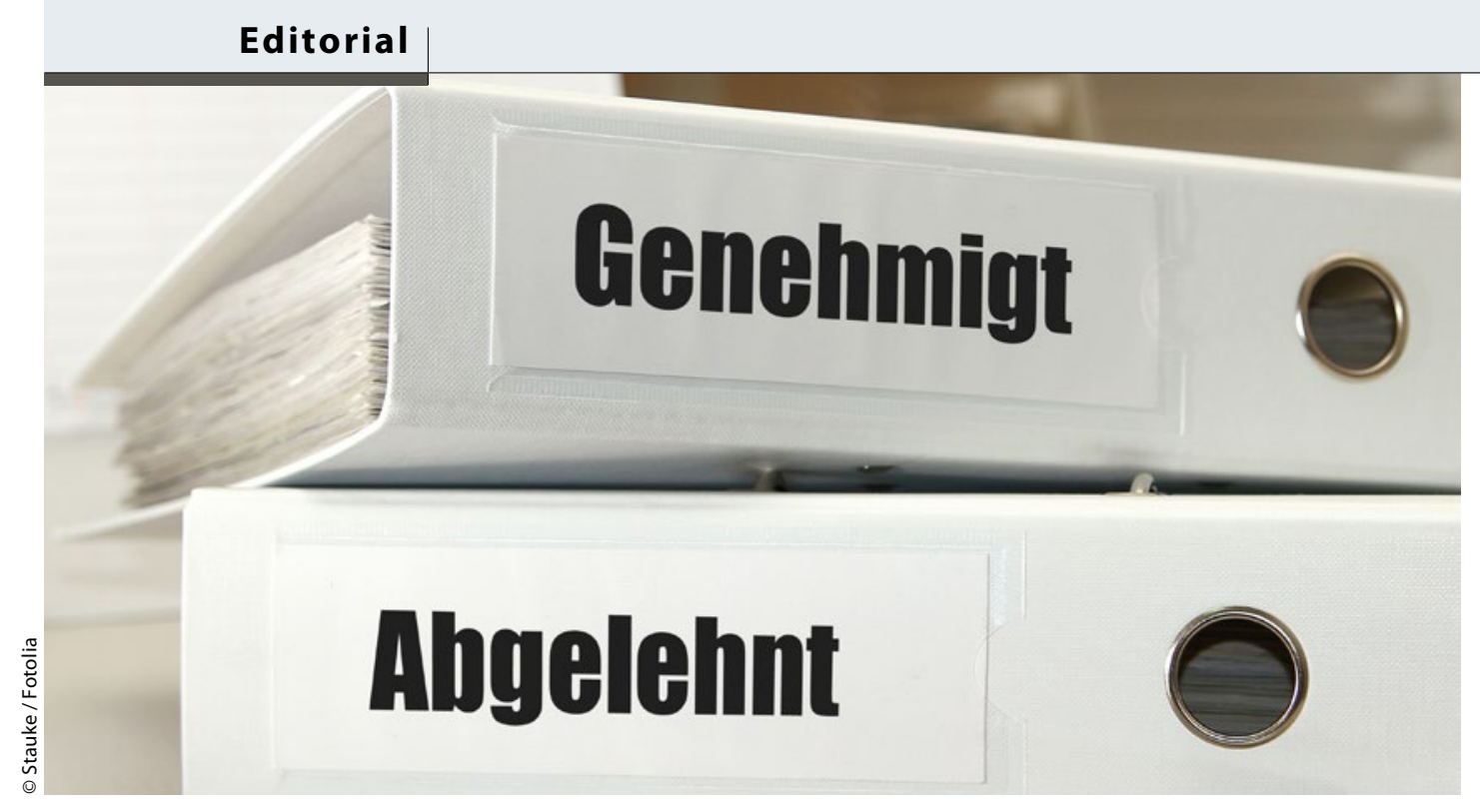

Von insgesamt 144 Nutzenbewertungen des G-BA wiesen 81 Wirkstoffe einen Zusatznutzen auf.

meist eine Polytherapie notwendig und die Erkrankung führt zu negativen Auswirkungen auf Lebensqualität, Berufstätigkeit sowie damit verbundene hohe indirekte Kosten (Tagestherapiekosten für neue innovative Medikamente hingegen liegen bei $3-8 €$ ). Für eine umfassende Behandlung dieser $\mathrm{Pa}$ tientengruppe ist der Zugang zu neuartigen AE notwendig. Einen Zusatznutzen zu belegen, scheitert allerdings bislang bei allen neuen AE an den Vorgaben, insbesondere der Definition einer „zweckmäßigen Vergleichstherapie“.

\section{Standardtherapie versagt bei einem Drittel}

In der Epileptologie werden noch immer placebokontrollierte Studien für die Zulassung gefordert, die „nur“ einen Nutzen, aber nicht einen Zusatznutzen gegenüber einem anderen Präparat nachweisen. Indirekte Vergleiche zweier Studien über den „Brückenkomparator" Placebo sind in der Regel nicht zielführend, aufgrund von Unterschieden in den untersuchten Patientenpopulationen und der jeweils angewandten Methodik. Daher akzeptiert der G-BA epileptologische Zulassungsstudien bisher nicht als Hinweis auf einen Zusatznutzen. Während etwa zwei Drittel aller Patienten mit den bereits verfügbaren Mitteln anfallsfrei werden, versagen in dem verbleibenden Drittel die zugelassenen Therapien. Bei Patienten mit einer langjährigen und refraktären Epilepsie gibt es anerkanntermaßen keine Standardtherapie oder gar standardisierte Abfolge weiterer Therapieformen mehr. Es müssen hochindividuelle Therapieentscheidungen getroffen werden, die die Ausprägung des Epilepsiesyndroms, die individuelle Krankheitshistorie und pharmakodynamische - wie pharmakokinetische - Aspekte berücksichtigen müssen [Hamer $\mathrm{H}$ et al. Nervenarzt 2016; 87: $351-2$ ].

Da es bislang nicht möglich ist, eine Subgruppe der medikamentös refraktären Epilepsiepatienten zu identifizieren, die auf das eine oder andere AE ansprechen wird, bleibt festzustellen, dass hier der Zusatznutzen ein Effekt ist, der sich individuell darstellt, aber in praxi nicht untersuchbar ist. Dies stellt auch den größten Kritikpunkt der DGfE an dem AMNOG in seiner jetzigen Form dar. Wenn die Regeln des IQWiG schon 1990 gegolten hätten, wäre in Deutschland keines der „neuen“ AE auf dem Markt geblieben, denn alle neuen AE sind im Headto-Head-Vergleich global allenfalls gleichwertig zu den alten Substanzen gewesen, selbst innovative Substanzen wie Levetiracetam [Brodie MJ et al. Neurology 2007; 68: 402 -8].

Die DGfE schlägt daher vor, placebokontrollierte Zulassungsstudien zunächst als Beleg für einen Zusatznutzen gelten zu lassen. Jedes neue AE sollte nach der Zulassung verfügbar werden, um dann an speziellen Zentren zu prüfen, wie gut die Chancen auf Anfallsreduktion oder Anfallsfreiheit sind. Zur Bestimmung des (individuellen) Zusatznutzens neuer AE sollten zunächst nur Experten an Epilepsiezentren oder Inhaber des Zertifikats „Epileptologie“ diese Substanzen verschreiben dürfen. Die Patienten sollten in eine industrieunabhängige Beobachtungsstudie eingeschlossen und strukturiert weiterverfolgt werden. Einige Jahre nach Einführung des Präparats würde dann durch den G-BA in Zusammenarbeit mit der Fachgesellschaft geprüft, ob die bisherigen Bewertungen der Substanz weiterhin Bestand haben und eine endgültige Bewertung des Zusatznutzens vorgenommen werden. Dieser Vorschlag der DGfE steht im Raum und könnte sehr gut auch für andere Fachgesellschaften gelten. Der G-BA kennt ihn und sollte nun reagieren.

Ihr

Thomas Mayer

Dr. med. Thomas Mayer

Sächsisches Epilepsiezentrum Radeberg Wachauerstr. 30, 01454 Radeberg E-Mail: t.mayer@kleinwachau.de 